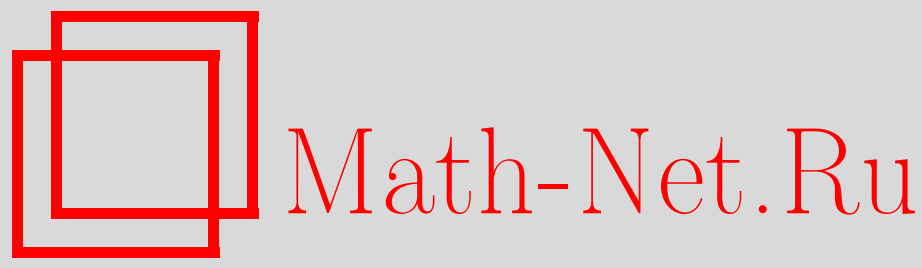

В. Ж. Сакбаев, Об усреднении квантовых динамических полугрупп, ТMФ, 2010, том 164, номер 3, 455-463

DOI: https://doi.org/10.4213/tmf6557

Использование Общероссийского математического портала Math-Net.Ru подразумевает, что вы прочитали и согласны с пользовательским соглашением http://www . mathnet.ru/rus/agreement

Параметры загрузки:

IP: 18.209 .158 .208

26 апреля 2023 г., 12:58:19

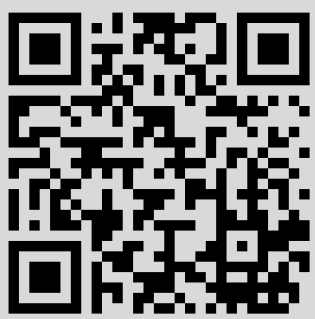




\title{
ФИЗИКА
}

Том 164, № 3

сентябрь, 2010

2010 г.

В. Ж. Сакбаев*

\section{ОБ УСРЕДНЕНИИ КВАНТОВЫХ ДИНАМИЧЕСКИХ ПОЛУГРУПП}

\begin{abstract}
С задачей Коши для уравнения Шредингера с разрывными вырождающимися коэффициентами в рамках метода эллиптической регуляризации связана последовательность регуляризованных задач Коши и соответствующих регуляризованных динамических полугрупп. Расходящаяся последовательность квантовых динамических полугрупп изучается как случайный процесс со значениями в пространстве квантовых состояний, определенный на измеримом пространстве параметров регуляризации с конечно-аддитивной мерой. Математическое ожидание рассматриваемых процессов, определенное интегралом Петтиса, задает семейство усредненных динамических преобразований. Исследовано полугрупповое свойство, свойства инъективности и сюръективности усредненных преобразований. Установлена возможность определения процесса по его математическому ожиданию в два различных момента времени и предложена процедура аппроксимации неизвестного начального состояния решениями конечного множества вариационных задач на компактных множествах.
\end{abstract}

Ключевые слова: стохастический процесс, конечно-аддитивная мера, квантовое состояние, динамическая полугруппа, наблюдаемость.

\section{1. ВВЕДЕНИЕ}

Явление вырождения гамильтониана на некотором подмножестве фазового или координатного пространства приводит к некорректности задачи Коши для уравнения эволюции как в классической [1], так и в квантовой [2] механике. Для определения динамики системы с вырожденным гамильтонианом используется метод эллиптической регуляризации вырожденного гамильтониана - динамическое преобразование пространства состояний такой системы определяется как предел последовательности регуляризованных динамических полугрупп, задаваемых регуляризованными гамильтонианами.

Процедурам предельного перехода для последовательностей динамических систем, определяемых дифференциальными уравнениями с малым параметром, и усреднения таких систем посвящена работа Боголюбова [3]. В монографии [4] развита

* Московский физико-технический институт, Долгопрудный, Московская обл. E-mail: fumi2003@mail.ru 
теория усреднения на группах, в том числе банаховых средних. Настоящая работа посвящается применению операции усреднения по конечно-аддитивным мерам к квантовым системам с гамильтонианами, содержащими малый параметр.

Задача Коши для уравнения Шредингера с вырожденным гамильтонианом

$$
\begin{aligned}
i \frac{d}{d t} u(t) & =\mathbf{L} u(t), & t & >0, \\
u(+0) & =u_{0}, & u_{0} & \in H=L_{2}(R)
\end{aligned}
$$

(здесь $\mathbf{L}$ - симметрический плотно определенный оператор в пространстве $H$ ) изучается методом эллиптической регуляризации. Исследуется последовательность регуляризованных задач Коши с начальным условием (2) для уравнений Шредингера

$$
i \frac{d}{d t} u(t)=\mathbf{L}_{\varepsilon} u(t), \quad t>0, \quad \varepsilon \in E \equiv(0,1)
$$

где при каждом $\varepsilon \in E$ оператор $\mathbf{L}_{\varepsilon}$ самосопряжен и равномерно эллиптичен, а при $\varepsilon \rightarrow 0$ последовательность операторов $\left\{\mathbf{L}_{\varepsilon}\right\}$ аппроксимирует оператор $\mathbf{L}$.

В качестве примера вырожденного симметрического оператора может быть предложен оператор $\mathbf{L}$, заданный на максимальной области определения в пространстве $H=L_{2}(R)$ дифференциальным выражением второго порядка

$$
\mathbf{L} v(x)=\frac{\partial}{\partial x}\left(g(x) \frac{\partial}{\partial x} v(x)\right)+\frac{i}{2}\left(a(x) \frac{\partial}{\partial x} v(x)+\frac{\partial}{\partial x}(a(x) v(x))\right)
$$

где $g=\chi_{\mathbb{R}_{-}}$есть характеристическая функция полупрямой $\mathbb{R}_{-}=(-\infty, 0)$, а вещественнозначная функция $a=\alpha \chi_{\mathbb{R}_{+}}$пропорциональна характеристической функции полупрямой $\mathbb{R}_{+}=(0,+\infty)$. Примером регуляризации служит семейство операторов $\mathbf{L}_{\varepsilon}$, заданных на максимальной области определения в пространстве $H$ дифференциальным выражением вида (4) с функцией $g_{\varepsilon}=g+\varepsilon, \varepsilon \in E$, вместо $g$ (см. работу [5]).

При каждом значении $\varepsilon \in E$ оператор $\mathbf{L}_{\varepsilon}$ генерирует регуляризованную унитарную полугруппу $\mathbf{U}_{\mathbf{L}_{\varepsilon}}(t)=e^{-i \mathbf{L}_{\varepsilon} t}, t>0$, в пространстве $H$ и определяет регуляризованное решение $u_{\varepsilon}\left(t, u_{0}\right)=\mathbf{U}_{\mathbf{L}_{\varepsilon}}(t) u_{0}$. В настоящей работе мы исследуем случай (подобный приведенному в примере после равенства (4)), когда оператор $\mathbf{L}$ является максимальным симметрическим оператором, а оператор $-i \mathbf{L}$ не является генератором сжимающей полугруппы, что в терминах индексов дефекта оператора $\mathbf{L}$ эквивалентно условиям

$$
\operatorname{dim}\left(\operatorname{Ker}\left(\mathbf{L}^{*}-i \mathbf{I}\right)\right)=0, \quad \operatorname{dim}\left(\operatorname{Ker}\left(\mathbf{L}^{*}+i \mathbf{I}\right)\right)>0
$$

В работе [5] доказано, что при выполнении условия (5) оператор $i \mathbf{L}$ генерирует изометрическую полугруппу $\mathbf{U}_{-\mathbf{L}}(t)=e^{i \mathbf{L} t}, t>0$, в пространстве $H$, и сопряженный оператор $-i \mathbf{L}^{*}$ генерирует сжимающую полугруппу $\mathbf{U}_{\mathbf{L}^{*}}(t)=e^{-i \mathbf{L}^{*} t}, t>0$, и справедливо следующее предложение. 
ПреДЛОЖЕНИЕ 1. Пусть выполнены условия (5). Тогда при каждом $t>0$ пространство $H$ допускает ортогональное разложение $H=H_{0}(t) \oplus H_{1}(t)$, где $H_{0}(t)=$ $\overline{\operatorname{Im}(\mathbf{U}-\mathbf{L}(t))}$ и $H_{1}(t)=\operatorname{Ker}\left(\mathbf{U}_{\mathbf{L}^{*}}(t)\right)$, таким образом, что для любого $u_{0} \in H_{1}(t)$ последовательность регуляризованных решениц $\left\{u_{\varepsilon}\left(t, u_{0}\right)\right\}$ при $\varepsilon \rightarrow 0$ слабо сходится в $H \kappa$ нулю, а при любом $u_{0} \in H_{0}(t)$ справедливо равенство $\lim _{\varepsilon \rightarrow 0} \| u_{\varepsilon}\left(t, u_{0}\right)-$ $\mathbf{U}_{\mathbf{L}^{*}}(t) u_{0} \|_{H}=0$.

Квантовые состояния. Пусть $B(H)$ есть банахово пространство линейных ограниченных операторов в пространстве $H$ и $B^{*}(H)$ - его сопряженное. Далее через $\Sigma(H)=S_{1}\left(B^{*}(H)\right) \cap B_{+}^{*}(H)$ обозначается множество квантовых состояний части единичной сферы пространства $B^{*}(H)$ (здесь и далее через $S_{1}(X)$ обозначается единичная сфера в банаховом пространстве $X$ ), лежащей в положительном конусе $B_{+}^{*}(H)$ функционалов из $B^{*}(H)$, принимающих неотрицательные значения на положительных операторах из пространства $B(H)$ [6]. Через $\Sigma_{\mathrm{n}}(H)$ обозначим множество нормальных квантовых состояний - функционалов из $\Sigma(H)$, непрерывных не только по норме, но и в сильной операторной топологии. Через $\Sigma_{\mathrm{p}}(H)$ будем обозначать множество чистых квантовых состояний - множество крайних точек пространства $\Sigma_{\mathrm{n}}(H)$, задаваемых проекторами на одномерные подпространства пространства $H$ [6]. Чистое квантовое состояние, отвечающее проектору на единичный вектор $u \in H$, задает на пространстве $B(H)$ линейный непрерывный функционал $\rho_{u}:\left\langle\rho_{u}, \mathbf{A}\right\rangle=(u, \mathbf{A} u)_{H}$.

Чтобы проанализировать свойства расходящейся ограниченной последовательности решений $\left\{u_{\varepsilon}\left(t, u_{0}\right)\right\}$, мы рассмотрим соответствующую ей последовательность регуляризованных операторов плотности $\left\{\rho_{\varepsilon}\left(t, \rho_{u_{0}}\right), t>0, \varepsilon \in E, \varepsilon \rightarrow 0\right\}$, где при каждом $\varepsilon \in E$ функция $\rho_{\varepsilon}\left(t, \rho_{0}\right): \mathbb{R}_{+} \rightarrow B^{*}(H)$ определена равенством $\left\langle\rho_{\varepsilon}\left(t, \rho_{0}\right), \mathbf{A}\right\rangle=$ $\left\langle\rho_{0}, \mathbf{U}_{\mathbf{L}_{\varepsilon}}(-t) \mathbf{A U}_{\mathbf{L}_{\varepsilon}}(t)\right\rangle$. Исследуем также последовательность регуляризованных динамических полугрупп $\left\{T_{\varepsilon}(t), t>0\right\}_{\varepsilon \in E, \varepsilon \rightarrow 0}$ в пространстве $B^{*}(H)$, каждая из которых определяется равенством $T_{\varepsilon}(t) \rho_{0}=\rho_{\varepsilon}\left(t, \rho_{0}\right)$.

В работе [7] установлено, что необходимым и достаточным условием секвенциальной компактности множества значений последовательности $\left\{\rho_{\varepsilon}\left(t, \rho_{u_{0}}\right)\right\}$ в $*$-слабой топологии пространства $B^{*}(H)$ является включение $u_{0} \in H_{0}(t)$. В случае $u_{0} \notin$ $H_{0}(t)$ определим для каждого оператора $\mathbf{A} \in B(H)$ множество $G_{A}\left(t, u_{0}\right)=\{r \in$ $\left.\Sigma(H):\langle r, \mathbf{A}\rangle \in L s_{\varepsilon \rightarrow 0}\left\langle\rho_{\varepsilon}\left(t, u_{0}\right), \mathbf{A}\right\rangle\right\}$, где $L s_{\varepsilon \rightarrow 0}\left\langle\rho_{\varepsilon}\left(t, u_{0}\right), \mathbf{A}\right\rangle-$ множество частичных пределов числовой функции $\left\langle\rho_{\varepsilon}\left(t, u_{0}\right), \mathbf{A}\right\rangle, \varepsilon \in E$, при $\varepsilon \rightarrow 0$. Согласно работе [7] множество $\Phi\left(t, u_{0}\right)=\bigcap_{\mathbf{A} \in B(H)} G_{\mathbf{A}}\left(t, u_{0}\right)$ выпукло, замкнуто в *-слабой топологии пространства $B^{*}(H)$ и удовлетворяет равенству $\left\langle\Phi\left(t, u_{0}\right), \mathbf{A}\right\rangle=L s_{\varepsilon \rightarrow 0}\left\langle\rho_{\varepsilon}\left(t, u_{0}\right), \mathbf{A}\right\rangle$ при любом $\mathbf{A} \in B(H)$.

Случайный процесс. Рассмотрим $\sigma$-алгебру $2^{E}$ всех подмножеств множества параметров регуляризации $E$ и обозначим через $W(E)$ совокупность всех конечно-аддитивных мер, заданных на измеримом пространстве $\left(E, 2^{E}\right)$, неотрицательных, нормированных и удовлетворяющих следующему условию: мера любого множества $A \in 2^{E}$, замыкание которого не содержит предельную точку $e^{*}=0=\inf E$, равна нулю. Множество $W(E)$ есть непустое выпуклое подмножество в пересечении конуса неотрицательных элементов с единичной сферой в банаховом пространстве $\mathrm{ba}\left(E, 2^{E}\right)$ конечно-аддитивных мер на измеримом пространстве $\left(E, 2^{E}\right)[8]$. Множество $W(E)$, очевидно, замкнуто в $*$-слабой топологии пространства $\mathrm{ba}(E)$. Согласно 
теореме 33 из работы [8] множество крайних точек $\operatorname{Extr}(W(E))$ множества $W(E)$ представляет собой подмножество $W_{0}(E)$ двузначных мер $\mu \in W(E)$, принимающих только значения 0 и 1 на $\sigma$-алгебре $2^{E}$.

В работе [9] расходящаяся последовательность $\left\{\rho_{\varepsilon}\left(t, u_{0}\right)\right\}$ исследована как случайный процесс: семейство измеримых отображений (параметризованных переменной $t>0)$ измеримого пространства $\left(E, 2^{E}\right)$ с мерой $\mu \in W(E)$ в измеримое пространство - банахово пространство $B^{*}(H)$ с алгеброй цилиндрических подмножеств $\mathrm{Cyl}_{Y_{*}}(Y)$, порожденной конечными наборами функционалов из $B(H)$. Каждое семейство отображений $\left\{\rho_{\varepsilon}\left(t, u_{0}\right)\right\}$ удовлетворяет условию:

$$
\lim _{t \rightarrow+0} \sup _{\substack{\mathbf{A} \in B(H),\|\mathbf{A}\|_{B(H)}=1}}\left|\rho_{\varepsilon}\left(t, u_{0}\right)(\mathbf{A})-\rho_{u_{0}}(\mathbf{A})\right|=0
$$

при любом $\varepsilon \in E$ (т.е. процесс $\rho_{\varepsilon}\left(t, u_{0}\right)$ начинается в точке $\rho_{u_{0}}$ пространства $\left.B^{*}(H)\right)$. Математическое ожидание случайной величины $\rho_{\varepsilon}\left(t, \rho_{0}\right)$ при каждом $t>0$ определяется как интеграл Петтиса $\rho^{\mu}\left(t, \rho_{0}\right)=\int_{E} \rho_{\varepsilon}\left(t, \rho_{0}\right) d \mu$, т.е. линейный непрерывный функционал $\rho^{\mu}\left(t, \rho_{0}\right)$ на пространстве $B(H)$ задан равенством $\left\langle\rho^{\mu}\left(t, \rho_{0}\right), \mathbf{A}\right\rangle=$ $\int_{E}\left\langle\rho_{0}, \mathbf{U}_{\mathbf{L}_{\varepsilon}(t) *} \mathbf{A U}_{\mathbf{L}_{\varepsilon}(t)}\right\rangle[10]$.

В работе [9] многозначное отображение $\Phi\left(t, \rho_{u_{0}}\right)$ представлено как система непрерывных однозначных ветвей $\Phi\left(t, \rho_{u_{0}}\right)=\bigcup_{\mu \in W(E)} \rho^{\mu}\left(t, \rho_{u_{0}}\right)$. Каждая из ветвей $\rho^{\mu}\left(t, \rho_{u_{0}}\right)$ является математическим ожиданием случайного процесса $\rho_{\varepsilon}\left(t, \rho_{u_{0}}\right)$ на измеримом пространстве с мерой $\left(E, 2^{E}, \mu\right)$. Следовательно, каждой мере $\mu \in W(E)$ сопоставляется однопараметрическое семейство усредненных динамических преобразований $T^{\mu}(t), t>0$, пространства $B^{*}(H)$ таких, что $T^{\mu}(t) \rho_{0}=\rho^{\mu}\left(t, \rho_{0}\right)$, $\rho_{0} \in B^{*}(H)$. Очевидно, отображение $T^{\mu}(t)$ определено на пространстве $B^{*}(H)$ линейно и непрерывно, причем множество квантовых состояний $\Sigma(H)$ отображается им в себя. Нашей целью является изучение свойств однопарамертических семейств динамических преобразований $T^{\mu}(t), t>0$, пространства $B^{*}(H)$ в зависимости от свойств мер $\mu \in W(E)$ - полугруппового свойства, свойств инъективности и сюръективности, продолжающее исследования работы [11].

\section{2. СВОЙСТВА УСРЕДНЕННОГО ПРЕОБРАЗОВАНИЯ}

Напомним, что оператор $\mathbf{L}$ удовлетворяет предположению (5). Пусть $t>0$ и $H_{0}(t)=\overline{\operatorname{Im}\left(\mathbf{U}_{-\mathbf{L}}(t)\right)}$ и $H_{1}(t)=\operatorname{Ker}\left(\mathbf{U}_{\mathbf{L}^{*}}(t)\right)$. В работе [11] установлено, что при любом $\mu \in W(E)$ сужение $\left.T^{\mu}(t)\right|_{\Sigma\left(H_{0}(t)\right)}$ является биективным и задается равенством $T^{\mu}(t) \rho_{0}=e^{-i \mathbf{L}^{*} t} \rho_{0} e^{i \mathbf{L} t}, \rho_{0} \in \Sigma\left(H_{0}(t)\right)$.

Для описания свойств сужения $\left.T^{\mu}(t)\right|_{\Sigma\left(H_{1}(t)\right)}$ приведем доказанное в работе [11] утверждение.

Лемма 1. Пусть $t>0,\left\{e_{k}\right\}$ - базис пространства $H_{1}(t) u\left\{\varepsilon_{k}\right\}-$ бесконечно малая последовательность положительных чисел. Тогда каждому числу $m \in \mathbf{N}$ соответствует такая подпоследовательность $\left\{\varepsilon_{k}^{m}\right\}$ последовательности $\left\{\varepsilon_{k}\right\}$, что выполнены следующие утверждения:

1) для всех $k, l \in \overline{1, m}$ и любых $i, j \in \mathbf{N}, i \neq j$, выполнено неравенство

$$
\left|\left(\mathbf{U}_{\mathbf{L}_{\varepsilon_{i}^{m}}}(t) e_{k}, \mathbf{U}_{\mathbf{L}_{\varepsilon_{j}^{m}}}(t) e_{l}\right)\right|<2^{-(i+j+m)} ;
$$


2) для каждого $q \in \overline{\{1, m\}}$ последовательность $\left\{\varepsilon_{i}^{q}\right\}$ является подпоследовательностью $\left\{\varepsilon_{i}^{q-1}\right\}$, где $\varepsilon_{i}^{0}=\varepsilon_{i}, i \in \mathbf{N}$. Кроме того, существует мера $\mu \in W(E)$ такая, что для любого $m \in \mathbf{N}$ выполнено равенство $\mu\left(\bigcup_{k=1}^{\infty} \varepsilon_{k}^{m}\right)=1$.

Для каждого $t>0$ и выбранного базиса в пространстве $H_{1}(t)$ обозначим через $\mathcal{M}(t)$ множество мер, удовлетворяющих условию леммы 1 . Тогда из леммы 1 и результатов работы [11] вытекает следующее утверждение.

Теорема 1. Пусть $t>0 u \mu \in \mathcal{M}(t)$. Тогда образом выпуклого множества $\Sigma\left(H_{1}(t)\right)$ пространства $B^{*}(H)$ при отображении $T^{\mu}(t)$ является выпуклое множество $T_{t}^{\mu}\left(\Sigma\left(H_{1}(t)\right)\right)$ пространства $B(H)^{*}$. Образ множества $\Sigma_{p}\left(H_{1}(t)\right)$ крайних точек множества $\Sigma_{n}\left(H_{1}(t)\right)$ совпадает с множеством крайних точек образа $T^{\mu}(t)\left(\Sigma_{n}\left(H_{1}(t)\right)\right)$ :

$$
T^{\mu}(t)\left(\operatorname{Extr}\left(\Sigma_{n}\left(H_{1}(t)\right)\right)\right)=\operatorname{Extr}\left(T^{\mu}(t)\left(\Sigma_{n}\left(H_{1}(t)\right)\right)\right) .
$$

Существует обратное $\left.\kappa T^{\mu}(t)\right|_{\Sigma_{p}\left(H_{1}(t)\right)}$ отображсене $\Lambda^{\mu}(t)$.

Наблюдаемость случайного процесса. В работе [11] исследуется вопрос об определении начального состояния $\rho_{0}$ процесса $\rho_{\varepsilon}\left(t, \rho_{0}\right)$ на измеримом пространстве $\left(E, 2^{E}, \mu\right)$ с мерой $\mu \in \mathcal{M}(t)$ по набору значений его математического ожидания $\rho^{\mu}\left(t, \rho_{0}\right)$ на различных подмножествах алгебры $B(H)$.

Пусть $\left\{e_{j}^{0}\right\}$ и $\left\{e_{j}^{1}\right\}$ - ортонормированные базисы в подпространствах $H_{0}(t)$ и $H_{1}(t)$ соответственно, а $\mu \in \mathcal{M}(t)$. Пусть $H_{0,1}^{m}(t)$ есть $m$-мерное подпространство в пространстве $H_{0,1}(t)$, натянутое на первые $m$ базисных векторов. Для каждого $v_{0} \in$ $H_{0}^{m}(t)$ определим ортогональный проектор $\mathbf{P}_{\mathbf{U}_{\mathbf{L}^{*}}(t) v_{0}}$ (здесь и далее через $\mathbf{P}_{u}$ обозначается оператор ортогонального проектирования в пространстве $H$ на одномерную линейную оболочку вектора $u$ ), а для каждого вектора $v_{1} \in H_{1}^{m}(t)$ определим оператор $\mathbf{Q}_{m}\left(v_{1}\right)$ как предел (существующий в силу (6)) в сильной операторной топологии частичных сумм операторного ряда

$$
\mathbf{Q}_{m}\left(v_{1}\right)=\sum_{\varepsilon_{k}^{m} \in E^{m}} \mathbf{P}_{\mathbf{U}_{\mathbf{L}_{\varepsilon} m}^{m}(t) v_{1}} .
$$

Пользуясь попарной почти ортогональностью в смысле (6) членов ряда (7), можно показать, что для любого $m \in \mathbf{N}$ функция $\mathbf{Q}_{m}(\cdot)$ является непрерывным отображением $m$-мерной сферы $S_{1}\left(H_{1}^{m}(t)\right)$ в банахово пространство $B(H)$ (напомним, что $S_{1}(H)$ есть единичная сфера в пространстве $\left.H\right)$. При каждом $t>0$, каждом $m \in \mathbf{N}$ и каждом $\rho \in \Sigma(H)$ определим следующие функционалы:

$$
\begin{array}{ll}
\varphi_{0}^{t, m, \rho}(v)=\left\langle\rho, \mathbf{P}_{\mathbf{U}_{\mathbf{L}^{*}}(t) v}\right\rangle, & v \in S_{1}\left(H_{0}^{m}(t)\right) ; \\
\varphi_{1}^{t, m, \rho}(v)=\left\langle\rho, \mathbf{Q}_{m}(v)\right\rangle, & v \in S_{1}\left(H_{1}^{m}(t)\right) .
\end{array}
$$

Тогда при любых $t>0, m \in \mathbf{N}, s \in\{0,1\}$ и $\rho \in \Sigma(H)$ непрерывный функционал $\varphi_{s}^{t, m, \rho}$ достигает максимума на некотором векторе $v_{s}^{m}$ на $m$-мерной единичной cфepe $S_{1}\left(H_{s}^{m}(t)\right)$.

В разделе 4 работы [11] доказано, что для любых $\rho_{u_{0}} \in \Sigma_{p}(H)$, любых $m \in \mathbf{N}$ и $t>0$ справедливо равенство

$$
\varphi_{0}^{t, m, \rho^{\mu}\left(t, \rho_{u_{0}}\right)}(v)=\left\|\left(u_{0}, v\right)\right\|^{2},
$$


а для любых $\rho_{u_{0}} \in \Sigma_{p}\left(H_{1}(t)\right)$ справедлива оценка

$$
\varphi_{1}^{t, m, \rho^{\mu}\left(t, \rho_{u_{0}}\right)}(v)-\left|\left(u_{0}, v\right)\right|^{2}=o(1)
$$

при $m \rightarrow \infty$ (см. лемму 4.3 из работы [11]). Поэтому существуют пределы

$$
M_{s}(t) \equiv \lim _{m \rightarrow \infty} \sup _{v \in S_{1}\left(H_{s}^{m}(t)\right)} \varphi_{s}^{t, m, \rho^{\mu}\left(t, \rho_{0}\right)}(v)=\left\|\mathbf{P}_{H_{s}(t)} u_{0}\right\|^{2}, \quad s=0,1 .
$$

Тогда почти дословным повтрением хода рассуждений работы [11] доказывается

Теорема 2. Пусть $t>0$ и начальное состояние $\rho_{0}$ удовлетворяет одному из двух условий: $\rho_{0} \in \Sigma_{n}\left(H_{0}(t)\right)$ либо $\rho_{0} \in \Sigma_{n}\left(H_{1}(t)\right)$. Пусть мера $\mu \in \mathcal{M}(t)$. Тогда:

1) состояние $\rho_{0}$ является чистым, если и только если $\max \left\{M_{0}(t), M_{1}(t)\right\}=1$;

$2)$ если начальное состояние $\rho_{0}$ является векторным состоянием в подпространстве $H_{0}(t)$ (или $\left.H_{1}(t)\right)$ и $\left\{v_{0}^{m}\right\}$ (или $\left.\left\{v_{1}^{m}\right\}\right)$ есть последовательность векторов, доставляющих максимум функционалам $\varphi_{0}^{m, \rho^{\mu}\left(t, \rho_{0}\right)}\left(\right.$ или $\left.\varphi_{1}^{m, \rho^{\mu}\left(t, \rho_{0}\right)}\right)$, то последовательность чистых состояний $\left\{\rho_{v_{0}^{m}}\right\}$ (или $\left.\left\{\rho_{v_{1}^{m}}\right\}\right)$ сходится в пространстве $B^{*}(H)$ $\kappa$ начальному состоянию $\rho_{0}$, причем для определения начального состояния с заданной точностъю достаточно решить конечное число вариачионных задач.

Если вектор $u_{0}$ есть линейная комбинация $u_{00}(t)+u_{01}(t)$ векторов из подпространств $H_{0}(t)$ и $H_{1}(t)$ соответственно, а оператор $\mathbf{A} \in B(H)$, то справедливо равенство

$$
\begin{aligned}
\left\langle\rho_{\varepsilon}\left(t, u_{0}\right), \mathbf{A}\right\rangle= & \int_{E}\left(u_{\varepsilon}\left(t, u_{0}\right), \mathbf{A} u_{\varepsilon}\left(t, u_{0}\right)\right) d \mu= \\
= & \int_{E}\left[\left(u_{\varepsilon}\left(t, u_{00}\right), \mathbf{A} u_{\varepsilon}\left(t, u_{00}\right)\right)+\left(u_{\varepsilon}\left(t, u_{01}\right), \mathbf{A} u_{\varepsilon}\left(t, u_{01}\right)\right)+\right. \\
& \left.+2 \operatorname{Re}\left(u_{\varepsilon}\left(t, u_{01}\right), \mathbf{A} u_{\varepsilon}\left(t, u_{00}\right)\right)\right] d \mu .
\end{aligned}
$$

Согласно предложению 1, во-первых, предел $\mathbf{A} u_{\varepsilon}\left(t, u_{00}\right)$ при $\varepsilon \rightarrow 0$ существует и равен $\mathbf{A} \mathbf{U}_{-\mathbf{L}}(t)^{*} u_{00}: \lim _{\varepsilon \rightarrow 0}\left\|\mathbf{A} u_{\varepsilon}\left(t, u_{00}\right)-\mathbf{A} \mathbf{U}_{-\mathbf{L}}(t)^{*} u_{00}\right\|$. Во-вторых, в силу слабой в пространстве $H$ сходимости к нулю последовательности векторов $\left\{u_{\varepsilon}\left(t, u_{01}\right), \varepsilon \in\right.$ $E, \varepsilon \rightarrow 0\}$ справедливо равенство $\lim _{\varepsilon \rightarrow 0}\left(u_{\varepsilon}\left(t, u_{01}\right), \mathbf{A} u_{\varepsilon}\left(t, u_{00}\right)\right)=0$. Следовательно, $\int_{E}\left(u_{\varepsilon}\left(t, u_{01}\right), \mathbf{A} u_{\varepsilon}\left(t, u_{00}\right)\right) d \mu=0$ и

$$
\left\langle\rho_{\varepsilon}\left(t, u_{0}\right), \mathbf{A}\right\rangle=\int_{E}\left(u_{\varepsilon}\left(t, u_{00}\right), \mathbf{A} u_{\varepsilon}\left(t, u_{00}\right)\right) d \mu+\int_{E}\left(u_{\varepsilon}\left(t, u_{01}\right), \mathbf{A} u_{\varepsilon}\left(t, u_{01}\right)\right) d \mu .
$$

Итак, получено следующее утверждение о неразличимости начальных состояний с помощью измерений средних значений наблюдаемых (11) в один момент времени $t>0$.

ПрЕДЛОЖЕНИЕ 2. Пусть $t>0$ и вектор состояния $u_{0} \in S_{1}(H)$ имеет проекции $u_{00}$ и $u_{01}$ на подпространства $H_{0}(t)$ и $H_{1}(t)$ соответственно. Тогда для любъх $\mu \in W(E)$ функииональ $\rho^{\mu}\left(t, u_{00}+e^{i \alpha} u_{01}\right)$ совпадают при всех $\alpha \in[0,2 \pi]$.

Лемма 2. Пусть $\rho_{0}=\rho_{u_{0}} \in \Sigma_{p}(H), s \in\{0,1\}$ и обе проекиии вектора $u_{0}$ на подпространства $H_{0}(t)$ и $H_{1}(t)$ нетривиальны. Тогда, если последовательность $\left\{v_{0}^{m}\right\}$ единичных векторов пространства $H_{0}(t)$ удовлетворяет равенству

$$
\sup _{v \in S^{1}\left(H_{0}^{m}(t)\right)} \varphi_{0}^{t, m, \rho^{\mu}\left(t, \rho_{0}\right)}(v)=\varphi_{0}^{t, m, \rho^{\mu}\left(t, \rho_{0}\right)}\left(v_{0}^{m}\right)
$$


при каждом $m \in \mathbf{N}$, то последовательность $\left\{v_{0}^{m}\right\}$ компактна в $H$, а любой ее частичный предел $v_{0}^{*}$ есть единичный вектор из одномерной линейной оболочки проекции $u_{00}$ вектора $u_{0}$ на подпространство $H_{0}(t)$.

Основой доказательства леммы 2 служит равенство (9). Пусть выполнены условия леммы 2 и $v_{0}^{*} \in H_{0}(t)$ - один из частичных пределов последовательности элементов $\left\{v_{0}^{m}\right\}$, доставляющих максимум функционалам $\varphi_{0}^{t, m, \rho^{\mu}\left(t, \rho_{0}\right)}$. При каждом $m \in \mathbf{N}$ и каждом $t>0$ определим функционал

$\psi^{t, m, \rho^{\mu}\left(t, \rho_{0}\right)}(v)=\varphi_{1}^{t, m, \rho^{\mu}\left(t, \rho_{0}\right)}(v)-M_{0}(t)\left(\mathbf{U}_{\mathbf{L}^{*}}(t) v_{0}^{*}, \mathbf{Q}_{m}(v) \mathbf{U}_{\mathbf{L}^{*}}(t) v_{0}^{*}\right), \quad v \in S_{1}\left(H_{1}^{m}(t)\right)$.

ЛЕмма 3. Пусть выполнены условия леммы 2. Тогда при каждых $t>0$ u $m \in \mathbf{N}$ функционал $\psi^{t, m, \rho^{\mu}\left(t, \rho_{0}\right)}(v)$ не зависит от выбора частичного предела $v_{0}^{*}$ u достигает максимума $M^{m}(t)$ в некоторой точке $v_{1}^{m} \in S_{1}\left(H_{1}^{m}(t)\right)$. При этом последовательность $\left\{M^{m}(t)\right\}$ сходится, и ее предел $M(t)$ удовлетворяет равенству $M(t)=1-M_{0}(t)$, а последовательность $\left\{v_{1}^{m}\right\}$ компактна в пространстве $H$, и любой ее частичный предел $v_{1}^{*}$ допускает представление $v_{1}^{*}=(M(t))^{-1 / 2} e^{-i \beta} u_{01}$ при некотором $\beta \in[0,2 \pi]$.

Лемма 3 может быть доказана на основании равенств (10) и (11).

Согласно лемме 3 равенство $M_{0}(t)+M(t)=1$ необходимо для включения $\rho_{0} \in$ $\Sigma_{p}(H)$, но недостаточно, ибо если $v_{0} \in S_{1}\left(H_{0}(t)\right)$ и $v_{1} \in S_{1}\left(H_{1}(t)\right)$, то для смешанного начального состояния $\rho_{0}=M_{0}(t) \rho_{v_{0}}+M(t) \rho_{v_{1}}$ выполнены равенства

$$
\lim _{m \rightarrow \infty} \sup \left(\varphi_{0}^{t, m, \rho^{\mu}\left(t, \rho_{0}\right)}(v)\right)=M_{0}(t)
$$

И

$$
\lim _{m \rightarrow \infty} \sup \left(\psi^{t, m, \rho^{\mu}\left(t, \rho_{0}\right)}(v)\right)=M(t) .
$$

Действительно, для любого $\mathbf{A} \in B(H)$ выполняется равенство $\left\langle\rho^{\mu}\left(t, \rho_{0}\right), \mathbf{A}\right\rangle=$ $M_{0}(t)\left\langle\rho^{\mu}\left(t, \rho_{v_{0}}\right), \mathbf{A}\right\rangle+M(t)\left\langle\rho^{\mu}\left(t, \rho_{v_{1}}\right), \mathbf{A}\right\rangle$. Тогда требуемое равенство следует из формул (11) и (8).

Покажем, что для однозначного определения динамики математического ожидания $\rho^{\mu}\left(t, \rho_{u_{0}}\right)$ процесса $\rho_{\varepsilon}\left(t, \rho_{u_{0}}\right)$ с чистым начальным состоянием $\rho_{u_{0}}$ достаточно знать значения математического ожидания в два различных момента времени.

Пусть при заданном $t>0$ значения $M_{0}(t), M(t)$ удовлетворяют равенству $M_{0}(t)+$ $M(t)=1$ и элементы $v_{0}^{*}$ и $v_{1}^{*}$ суть частичные пределы последовательностей $\left\{v_{0}^{m}\right\}$ и $\left\{v_{1}^{m}\right\}$ из лемм 2 и 3 . Если $M_{0}(t)=0$ (либо $M(t)=0$ ), то начальный вектор $u_{0}$ целиком лежит в подпространстве $H_{1}(t)$ (либо $H_{0}(t)$ ), и согласно теореме 2 начальное состояние $\rho_{u_{0}}$ совпадает с $\rho_{v_{1}^{*}}$ (либо с $\rho_{v_{0}^{*}}$ ). Исследуем случай, когда для выбранного момента времени $t>0$ выполняется соотношение $M_{0}(t) \in(0,1)$. Положим $H_{0}=\bigcap_{\tau>0} H_{0}(\tau)$, определим по вектору $v_{0}^{*}(t) \in H_{0}(t)$ число

$$
T^{*}=\inf \left\{s \geqslant t: \mathbf{U}_{\mathbf{L}^{*}}(s-t) v_{0}^{*}(t) \in H_{0}\right\}
$$

и положим $T^{*}=+\infty$, если $\mathbf{U}_{\mathbf{L}^{*}}(s) v_{0}^{*}(t) \notin H_{0}$ при любом $s>0$. Тогда существует такое $t_{1} \in\left(0, T^{*}\right)$, что $M_{0}\left(t_{1}\right) \neq M_{0}(t)$ (см. предложение 1 , а также работу [7]).

Так же, как и лемма 1 , с помошью метода математической индукции доказывается 
Лемма 4. Пусть $t>0,\left\{t_{j}, j \in \mathbf{N}\right\}$ - некоторая нумерация положительных рациональных чисел $u t_{0}=t$. Пусть в пространствах $H_{1}\left(t_{j}\right)$ выбраны ортонормированные базисы $\left\{e_{k}^{j}\right\}$ u $\left\{\varepsilon_{k}\right\}$ - бесконечно малая последовательность положительных чисел. Тогда каждому числу $m \in \mathbf{N}$ соответствует такая подпоследовательность $\left\{\varepsilon_{k}^{m}\right\}$ последовательности $\left\{\varepsilon_{k}\right\}$, что выполнены следующие утверждения:

1) для всех $k, l \in \overline{1, m}$, всех $p \in \overline{0, m}$ и любых $i, j \in\{0,1,2, \ldots\}, i \neq j$, выполнено неравенство

$$
\left|\left(\mathbf{U}_{\mathbf{L}_{\varepsilon_{i}^{m}}}\left(t_{p}\right) e_{k}^{p}, \mathbf{U}_{\mathbf{L}_{\varepsilon_{j}^{m}}}\left(t_{p}\right) e_{l}^{p}\right)\right|<2^{-(i+j+m)} ;
$$

2) для каждого $q \in \overline{\{1, m\}}$ последовательность $\left\{\varepsilon_{i}^{q}\right\}$ является подпоследовательностью $\left\{\varepsilon_{i}^{q-1}\right\}$, где $\varepsilon_{i}^{0}=\varepsilon_{i}, i \in \mathbf{N}$. Кроме того, существует мера $\nu \in W(E)$ такая, что для любого $m \in \mathbf{N}$ выполнено равенство $\nu\left(\bigcup_{k=1}^{\infty} \varepsilon_{k}^{m}\right)=1$.

Пусть $\nu$ - мера, существование которой установлено леммой 4. Тогда для каждого числа $t_{j}, j \in\{0,1,2, \ldots\}$, и меры $\nu$ справедливы утверждения лемм 2 и 3 . Поэтому, если $\rho_{0} \in \Sigma_{p}(H)$, то существуют частичные пределы $v_{0}^{*}\left(t_{j}\right), v_{1}^{*}\left(t_{j}\right)$ последовательностей, элементы которых реализуют максимумы функционалов $\varphi_{0}^{t_{j}, m, \rho^{\nu}\left(t_{j}, \rho_{0}\right)}$, $\psi^{t_{j}, m, \rho^{\nu}\left(t_{j}, \rho_{0}\right)}$ соответственно, и существуют пределы $M_{0}\left(t_{j}\right)=\lim _{m \rightarrow \infty} M_{0}^{m}\left(t_{j}\right)$ и $M\left(t_{j}\right)=\lim _{m \rightarrow \infty} M^{m}\left(t_{j}\right)$. В пространстве $B^{*}(H)$ определим кривые

$$
\mathcal{C}\left(t_{j}\right)=\left\{\rho_{\left(M_{0}\left(t_{j}\right)\right)^{1 / 2} v_{0}^{*}\left(t_{j}\right)+e^{i \alpha}\left(M\left(t_{j}\right)\right)^{1 / 2} v_{1}^{*}\left(t_{j}\right)}, \alpha \in[0,2 \pi)\right\}, \quad j \in \mathbf{N} .
$$

ТЕОРема 3. Пусть $t>0$ и $\rho_{0}-$ нормальное состояние.

1. Если состояние $\rho_{0}$ чистое, то выполнень равенства

$$
M_{0}(t)+M(t)=1, \quad \lim _{m \rightarrow \infty} \sup _{v \in S_{1}\left(H_{0}(t)\right)}\left[\varphi_{0}^{t, m, \rho^{\mu}\left(t, \rho_{0}\right)}(v)-M_{0}(t)\left|\left(v_{0}^{*}, v\right)\right|^{2}\right]=0
$$

и включение $\rho_{0} \in \mathcal{C}(t)$.

2. Если равенство $M_{0}(t)+M(t)=1$ выполнено и $M_{0}(t) \in(0,1)$, то существует такое рациональное число $t_{j} \in\left(0, T^{*}\right)$, что $M_{0}\left(t_{j}\right) \neq M_{0}(t)$. Кроме того, пусть $\lim _{m \rightarrow \infty} \sup _{v \in S_{1}\left(H_{0}(t)\right)}\left[\varphi_{0}^{t, m, \rho^{\mu}\left(t, \rho_{0}\right)}(v)-M_{0}(t)\left|\left(v_{0}^{*}, v\right)\right|^{2}\right]=0$. Тогда для включения $\rho_{0} \in \Sigma_{p}(H)$ достаточно, чтобы $M_{0}\left(t_{j}\right)+M\left(t_{j}\right)=1$. В этом случае кривые $\mathcal{C}(t)$ u $\mathcal{C}\left(t_{j}\right)$ имеют единственную общую точку, которая и является начальным состоянием $\rho_{0}$.

Математическое ожидание $T^{\nu}(t)$ случайного процесса $T_{\varepsilon}(t)$ как функции переменной $t$ не является однопараметрической полугруппой преобразований банахова пространства $B^{*}(H): T^{\nu}(t+\Delta t) \rho_{u_{0}} \neq T^{\nu}(\Delta t)\left(T^{\nu}(t) \rho_{u_{0}}\right)$, ибо существуют состояния $\rho_{u_{00}+e^{i \alpha} u_{01}}, \alpha \in[0,2 \pi]$, имеющие согласно предложению 2 общий образ при отображении $T^{\nu}(t)$, которые в силу теоремы 3 имеют различные образы при отображении $T^{\nu}(t+\Delta t)$ с некоторым $\Delta t>0$.

Благодарности. Работа выполнена при поддержке РФФИ (гранты № 09-0100265, 10-01-00395), Федеральной целевой программы "Научные и научно-педагогические кадры инновационной России" на 2009-2013 гг. и Аналитической ведомственной целевой программы "Развитие научного потенциала высшей школы" (проект № $2.1 .1 / 500)$. 


\section{Список литературы}

[1] В. В. Козлов, Тепловое равновесие по Гиббсу и Пуанкаре, Современная математика, РХД, М., Ижевск, 2002.

[2] L. Accardi, Y.G. Lu, I. V. Volovich, Quantum Theory and its Stochastic Limit, Texts Monogr. Phys., Springer, Berlin, 2001.

[3] Н. Н. Боголюбов, О некоторых статистических методах в математической физике, АН УССР, Киев, 1945.

[4] Н. Н. Боголюбов, "О некоторых эргодических свойствах непрерывных групп преобразований”, Избранные труды. т. I, Наукова думка, Киев, 1969, 561-569.

[5] В. Ж. Сакбаев, Тр. МИАН, 261 (2008), 258-267.

[6] У. Браттели, Д. Робинсон, Операторные алгебры и квантовая статистическая механика, Мир, М., 1982.

[7] В. Ж. Сакбаев, Ж. вычисл. матем. и матем. физ., 46:4 (2006), 683-699.

[8] В. С. Варадарайн, Матем. сб., 55(97):1 (1961), 35-100.

[9] Г. Г. Амосов, В. Ж. Сакбаев, Трудъ СамГУ, 8:1(67) (2008), 479-494.

[10] Р. Эдвардс, Функииональный анализ, Мир, М., 1969.

[11] В. Ж. Сакбаев, Труды МФТИ, 1:4 (2009), 126-147. 